\title{
Prestrain-Induced Diverse Creep Behaviour of Superalloy 718
}

\author{
S. Srinivas and M.C. Pandey \\ Defence Metallurgical Research Laboratory \\ Kanchanbagh, Hyderabad-500, India
}

\begin{abstract}
The influence of room-temperature prestraining on the creep behaviour of supcralloy 718 has been studied at $650^{\circ} \mathrm{C}$ in the stress range of $650-810 \mathrm{MPa}$. Both creep strengthening and weakening effects were observed depending on the microstructure of the alloy. Prestraining of the alloy with delta phase led to creep weakening resulting in poor creep properties. Premature nucleation of voids during creep, die to strain localization at the delta phase-matrix interface during room-temperature prestraining, led to creep weakening in the prestrained alloy. Prestraining of the alloy without delta phase, on the other hand, enhanced creep strength. The beneficial role of prestraining has been elucidated by considering the increase in back stress due to dislocation-dislocation interaction. In this case, the creep curves displayed true steady state at all prestrain levels, while non-prestrained specimens exhibited usual minimum creep rate. Also, the amount of primary creep strain increased and the fraction of time spend during tertiary stage decreased with increasing the prestrain level. The creep curves of the alloy have been analyzed and are explained in terms of the cavitational process and the change of fracture behaviour with prestraining and microstructural variation. The results are supplemented by optical, scanning and transmission electron microscopy. These findings have clear technological implications since the alloy may be plastically strained either during fabrication or incrementally throughout life, whereas designs are based on the creep data obtained on non-prestrained material.
\end{abstract}

\footnotetext{
Superalloys $718,625,706$ and Various Derivatives Ldited by E.A. Loria 